\title{
NS Fivebranes and Type 0 Dualities *
}

\author{
Ben $\mathrm{Craps}^{\dagger}$ and Frederik Roose
}

Instituut voor theoretische fysica

Katholieke Universiteit Leuven, B-3001_Leuven, Belgium

E-mail: Ben.Craps, Frederik. Roose@fys.kuleuven ac. be'

ABSTRACT: The massless degrees of freedom of type 0 NS5-branes are derived. A non-chiral, purely bosonic spectrum is found in both type $0 \mathrm{~A}$ and $0 \mathrm{~B}$. This non-chirality is confirmed by a one-loop computation in the bulk. Some puzzles concerning type 0B S-duality are pointed out in this context. An interpretation of the spectra in terms of "type 0 little strings" is proposed. This contribution is based on Ref. [1].

\section{Introduction}

Type 0 string theories [2] have recently enjoyed a renewed interest. A conjecture by Bergman

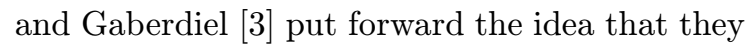
might be supersymmetry breaking orbifolds of M-theory, thus including them in the web of string dualities. Part of the conjecture says that type OB string theory exhibits S-duality and type 0 theories contain non-perturbative fermionic states.

D-branes, whose dynamics is governed by the open strings ending on them, can be studied in perturbative string theory. In contrast, a study of the low-energy spectrum on solitonic (NS) fivebranes necessitates indirect methods, such as an analysis of the zero-modes of the corresponding classical supergravity solutions [i] (normalizable) zero-mode corresponds to a massless field on the fivebrane world-volume. Rather than doing this zero-mode analysis directly for type 0A/B NS5-branes, we first T-dualize them to Kaluza-Klein monopoles of type 0B/A. With this approach the zero-modes can be read off from the ten-dimensional bulk fields and the cohomology of the Euclidean Taub-NUT space. For both NS5-branes a non-chiral spectrum is found: an unrestricted antisymmetric tensor and six sca-

\footnotetext{
*Work supported by the European Commission TMR programme ERBFMRX-CT96-0045.

${ }^{\dagger}$ Aspirant FWO, Belgium
}

lars in $0 \mathrm{~A}$ and two gauge fields and four scalars in $0 \mathrm{~B}$.

In type IIA the spectrum of the NS5 is chiral and anomalous. The gravitational anomaly on the NS5 must be cancelled by anomaly inflow [5i]] from the bulk. The anomaly-cancelling bulk term [i] can be shown to be a string one-loop effect [i]1]. The fact that we find a non-anomalous spectrum on type 0A NS5-branes predicts that such an anomalous bulk term is not present in type $0 \mathrm{~A}$. Indeed, we will see that the type $0 \mathrm{~A}$ GSO projection does not allow a CP-odd oneloop term.

Having identified the rather unusual NS5brane spectrum in type 0B, one naturally wonders what the NS5 transforms into under the conjectured type 0B S-duality. Following Ref. [3] it seems natural to propose that the dual object is a bound state of an electric and a magnetic D5-brane. However, we will point out that this proposal gives rise to some puzzles.

Finally, an interpretation of the NS5-brane spectra in terms of "type 0 little strings" is proposed. This interpretation is suggested by the doubling of the gauge degrees of freedom on the NS-branes, which is reminiscent of (and, in fact, closely related to) the doubling of the RamondRamond spectrum in going from type II to type 0 . 


\section{Type 0 strings and D-branes}

In the Neveu-Schwarz-Ramond formulation, type II string theories are obtained by imposing independent GSO projections on the left and right moving parts. This amounts to keeping the following (left,right) sectors:

$$
\begin{array}{llll}
\text { IIB : } & (N S+, N S+), & (R+, R+), \\
& (N S+, R+) & , & (R+, N S+), \\
\text { IIA : } \begin{array}{ll}
(N S+, N S+) \\
(N S+, R-),
\end{array} & (R+, R-), \\
& (R+, N S+)
\end{array},
$$

where for instance $\mathrm{R} \pm$ is the Ramond sector projected with $P_{\mathrm{GSO}}=\left(1 \pm(-)^{F}\right) / 2, F$ being the world-sheet fermion number.

The type 0 string theories contain instead the following sectors:

$$
\begin{array}{lll}
0 \mathrm{~B}: \quad & (N S+, N S+),(R+, R+), \\
& (N S-, N S-),(R-, R-), \\
0 \mathrm{~A}: \quad(N S+, N S+),(R+, R-), \\
& (N S-, N S-),(R-, R+) .
\end{array}
$$

These theories do not contain bulk spacetime fermions, which would have to come from "mixed" (R,NS) sectors. The inclusion of the NS-NS sector with odd fermion numbers means that the closed string tachyon is not projected out. The third difference with type II theories is that the $\mathrm{R}-\mathrm{R}$ spectrum is doubled.

As discussed in the introduction, the spectrum of type 0 theories contains two $(p+1)$-form $\mathrm{R}-\mathrm{R}$ potentials for each even $(0 \mathrm{~A})$ or odd $(0 \mathrm{~B}) p$. We will denote these by $C_{p+1}$ and $C_{p+1}^{\prime}$, where the unprimed potentials are the ones present in type II. For our purposes, more convenient combinations are

$$
\left(C_{p+1}\right)_{ \pm}=\frac{1}{\sqrt{2}}\left(C_{p+1} \pm C_{p+1}^{\prime}\right)
$$

For $p=3$ these are the electric $(+)$ and magnetic $(-)$ potentials $[\overline{8}$. We will adopt this terminology also for other values of $p$.

There are four types of "elementary" D-bran-

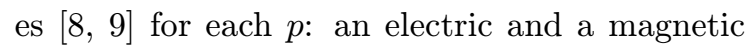
one (i.e. charged under $\left.\left(C_{p+1}\right)_{ \pm}\right)$, and the corresponding antibranes. In Ref. [in the interaction energy of two identical parallel $(p+1)$-branes was derived by computing the relevant cylinder diagram in the open string channel, analogously to the Polchinski computation in type II. Isolating, via modular transformation, the contributions due to the exchange of long-range fields in the closed string channel, it is found on the one hand that the tension of these branes is a factor $\sqrt{2}$ smaller than for type II branes. On the other hand, the R-R repulsive force between two like branes has the double strength of the graviton-

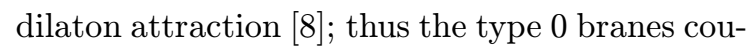
ple to the corresponding R-R potentials $\left(C_{p+1}\right)_{ \pm}$ with the same charge as the branes in type II couple to the potential $C_{p+1}$.

The open strings stretching between two like branes are bosons, just like the bulk fields of type 0 . However, fermions appear from strings be-

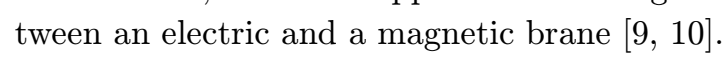

\section{NS fivebrane spectra}

In type II string theories the massless spectrum on NS5-branes can be derived via an analysis of the zero-modes of the classical supergravity NS5brane solutions [i]" We will find it convenient to do the analysis for the T-dual objects, since there the geometrical interpretation is manifest.

Under T-duality the type IIA/B NS5-branes are mapped to type IIB/A Kaluza-Klein (KK) monopoles. A KK monopole is described by a supergravity solution with six world-volume directions and a Euclidean Taub-NUT (ETN) metric in the transverse space. The massless fields on the KK monopole correspond to the zero modes of the bulk supergravity fields on ETN. The normalizable harmonic forms on ETN consist of one selfdual two-form. In the NS-NS sector there are three zero modes from broken translation invariance and one scalar from the NS two-form. These four scalars correspond to the translation zero modes on the corresponding NS5-branes. The R$\mathrm{R}$ fields give additional bosonic zero-modes: on the IIB KK monopole the R-R two-form gives rise to a scalar and the selfdual ${ }^{1} \mathrm{R}-\mathrm{R}$ four-form potential leads to a selfdual two-form; the IIA KK monopole has a vector coming from the R-R three-form. In addition there are fermionic zero modes from broken supersymmetry: two fermions

\footnotetext{
${ }^{1}$ By abuse of language we term the potential forms (anti-)selfdual, according to the (anti-)selfdual nature of their respective field strengths.
} 
with opposite chirality for the IIA KK monopole and of the same chirality for IIB.

All in all, this leads to an $\mathcal{N}=(2,0)$ tensor multiplet on the IIA NS5 and an $\mathcal{N}=(1,1)$ vector multiplet on the IIB NS5.

Repeating this analysis for type 0 NS5-branes, it is clear that the fermionic zero modes disappear (there are no fermions in the bulk). Nothing changes as far as the NS-NS zero modes are concerned, but from the R-R fields we find extra zero modes from the doubled R-R spectrum in the bulk. On the 0B NS5 there is an extra vector (compared to the IIB NS5), whereas the 0A NS5 gets an extra scalar and an anti-selfdual tensor. In particular the spectrum is non-chiral on both branes.

\section{Absence of anomalies}

At one string loop the type IIA tree level supergravity action is supplemented with the WessZumino type term $\int B \wedge X_{8}(R)$ coupling the NSNS two-form $B$ to four gravitons. $X_{8}$ is a quartic polynomial in the spacetime curvature two-form.

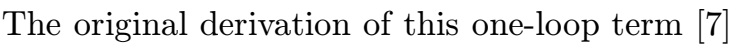
provided a non-trivial consistency check for sixdimensional heterotic-type II duality. Apart from that, this specific piece of the action is also responsible for gravitational anomaly cancellation on IIA NS fivebranes [i6̈].

Let us first highlight some aspects of the direct calculation in type II, which will enable us to draw conclusions on the analogous type 0 amplitude almost without effort. First, as the interaction contains the ten-dimensional $\epsilon$ symbol, only odd spin structures on the torus can contribute, i.e., either the left-moving or the right-moving fermions but not both, have to be in the odd spin structure. Furthermore, the even spin structures are summed over to achieve modular invariance. From a different perspective this may also be seen as performing the GSO projection on the closed string states in the loop. In a Hamiltonian framework the torus vacuum diagram is written as a trace over the closed string Hilbert space, which decomposes in four different sectors as in Eq. (2.1). Chiral traces over these sectors can be expressed in terms of traces over fermion sectors with periodic $(\mathrm{P})$ or antiperiodic $(\mathrm{A})$ boundary conditions in the $(\sigma, \tau)$ world-sheet directions:

$$
\begin{aligned}
\operatorname{Tr}_{(N S,-)} & =\frac{1}{2}((A, A)-(A, P)) ; \\
\operatorname{Tr}_{(N S,+)} & =\frac{1}{2}((A, A)+(A, P)) ; \\
\operatorname{Tr}_{(R,-)} & =\frac{1}{2}((P, A)-(P, P)) ; \\
\operatorname{Tr}_{(R,+)} & =\frac{1}{2}((P, A)+(P, P))
\end{aligned}
$$

Notice that the odd $(P, P)$ spin structure only occurs in the Ramond sector. From the GSOprojected spectrum in Eq. (2.12) only $(\mathrm{R}, \mathrm{NS})$ and $(\mathrm{R}, \mathrm{R})$ sectors contain (odd,even) pieces, yielding

$$
\frac{1}{4}((P, P),(A, A)+(A, P)+(P, A)) .
$$

The right-moving combination is modular invariant, and in fact vanishes as a consequence of the "abstruse identity". If one were to calculate the amplitude for one B-field and four gravitons, one would insert vertex operators in the appropriate pictures. Still, the right-moving even structures would be summed over as in Eq. (4.1). In contrast with the partition function however, in this case a non-vanishing result would be found so as not to contradict the alternative calculation in

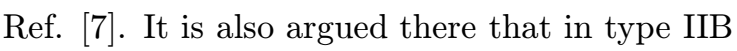
the (odd,even) and (even,odd) contributions cancel out whereas they add up in type IIA. So only in the latter is the probed interaction present.

This difference between type IIA and IIB could have been inferred from their respective NS fivebrane world-volume spectra, a chiral one in type IIA and a non-chiral one in type IIB. The chiral spectrum suffers from a gravitational anomaly in six dimensions, which is neatly cancelled by the standard anomaly inflow [s. from the bulk through the above derived coupling.

Let us repeat the argument for the one-loop correlator in type 0 string theories. Now it is only $(\mathrm{R}, \mathrm{R})$ sectors that contribute to the (odd,even) piece in the one-loop partition function, yielding

$$
\frac{1}{4}((P, P)-(P, P),(P, A))
$$

both for $0 \mathrm{~A}$ and $0 \mathrm{~B}$. As in type II one may now wish to insert vertex operators. However, it is obvious that the obligatory sum over spin structures as in Eq. (4.2i) now yields a vanishing result 
for the (odd,even) part by itself. Analogously, the (even,odd) part will vanish by itself. Whence the absence of the anomaly-cancelling term in both type $0 \mathrm{~A}$ and $0 \mathrm{~B}$.

Reversing the anomaly inflow argument in type $0 \mathrm{~A}$ the selfdual two-form must be supplemented with an anti-selfdual two-form, in order not to give rise to gravitational anomalies. This nicely agrees with the above derived unconstrained two-form on the NS fivebrane. From T-duality an additional vector (compared to the IIB NS5brane) has to be expected on the type 0B NS5brane.

\section{S-duality}

Strings In type IIB string theory the fundamental string is mapped to the D-string under S-duality. The Green-Schwarz light-cone formulation of the fundamental type IIB string involves eight spacetime bosonic fields and sixteen spacetime fermionic ones. All fermions transform in the $\mathbf{8}_{\mathbf{S}}$ representation of the transverse $\mathrm{SO}(8)$ rotation group. Upon quantization, their zero modes generate the 256 -fold degenerate groundstate, transforming in the $\left(\mathbf{8}_{\mathbf{V}}+\mathbf{8}_{\mathbf{C}}\right) \otimes\left(\mathbf{8}_{\mathbf{V}}+\mathbf{8}_{\mathbf{C}}\right)$ of $\mathrm{SO}(8)$.

The excitations of a D1-brane can be described in string perturbation theory by quantizing the open strings beginning and ending on it. Doing so, the NS sector gives eight massless bosons and the $\mathrm{R}$ sector sixteen massless fermions.

Note that these massless excitations of both the fundamental string and the D-string can be interpreted as Goldstone modes for broken translation invariance and broken supersymmetry.

It is believed that the IIB D-string at strong (fundamental) string coupling is the same object as a fundamental IIB string at weak coupling. The evidence for this conjecture is largely based on supersymmetry. For one thing, the ground state of the D-string is BPS, so that it can safely be followed to strong coupling and compared to the ground state of the weakly coupled fundamental string.

Type 0B string theory is not supersymmetric, which complicates the analysis of its strong coupling limit enormously. Let us nevertheless try to proceed.

The fundamental type $0 \mathrm{~B}$ string can be obtained from the fundamental type IIB string by performing a $(-1)^{F_{s}}$ orbifold, where $F_{s}$ is the spacetime fermion number. In the untwisted sector of this orbifold the massless states in the $\left(\mathbf{8}_{\mathbf{V}} \otimes \mathbf{8}_{\mathbf{V}}\right)+\left(\mathbf{8}_{\mathbf{C}} \otimes \mathbf{8}_{\mathbf{C}}\right)$ survive. The light modes in the twisted sector are a singlet tachyon and the extra massless R-R states in the $\mathbf{8}_{\mathbf{S}} \otimes \mathbf{8}_{\mathbf{S}}$.

Bergman and Gaberdiel conjectured [3] that the fundamental type 0B string should be S-dual to a bound state an electric and a magnetic D1brane. This proposal immediately raises two questions. First, when one quantizes the open strings on a coincident electric-magnetic D-string pair, one finds twice as many massless excitations as on a fundamental string: sixteen bosons and thirty-two fermions. This would lead to way too many states of the D-string pair compared to the fundamental string. How could the extra degrees of freedom disappear? Second, assuming that a way were found to get rid of the superfluous degrees of freedom, how does the $(-1)^{F_{s}}$ projection appear on the D-string pair?

As to the first question, in order to count modes one should really compactify the system: saying that one has two continua of states rather than one continuum does not make much sense. When a direction is compactified, the proposal in [3i] involves a monodromy on one side of the duality. It turns out that this monodromy eliminates the redundant would-be massless fermions.

As to the second question, the $(-1)^{F_{s}}$ projection might be related to a gauging of a $\mathbb{Z}_{2}$ subgroup of the gauge symmetry on the D-string pair. However, it looks hard to make this idea more precise.

Fivebranes Type IIB NS5-branes are S-dual to D5-branes. These objects have the same light excitations (the reduction of an $\mathcal{N}=1, \mathrm{D}=10$, $\mathrm{U}(1)$ vector multiplet to $\mathrm{D}=6$ ). As in the case of IIB strings, part of the spectrum is protected by supersymmetry.

Given the story for strings, it is tempting to propose [3] that the type 0B NS5-brane is Sdual to an electric-magnetic D5-brane pair. This raises two puzzles. 
First, how do the spectra match? Is the doubled gauge spectrum of type 0B NS5-branes at weak coupling related to the two gauge fields on the D5-brane pair? This is not clear, since to compare the two objects one has to take one of them to strong coupling, where it is unclear what happens. For one thing, the doubling of the gauge field on the type 0B NS5 (compared to the IIB NS5) is related to the doubled R-R spectrum of the bulk of type $0 \mathrm{~A}$, as shown in Section 3. The latter doubling is not expected to persist at strong coupling [i] zero modes on NS5-branes? We have not found any from a zero mode analysis of the bulk gravity theory, but this does not exclude that they could appear in a non-perturbative way. This would be analogous to the (not completely understood) case of the fundamental type 0 string, where it looks impossible to interpret the fermion zero modes as zero modes of bulk fields.

Second, do type 0B NS5-branes carry anomalous gravitational couplings, which are known to be carried by type 0B D-branes [10 01 ? Since these couplings are related to anomalies and thus to topology, we expect that their presence or absence could be invariant under continuous changes of the coupling constant. If this is the case, answering this question could provide a test of type OB S-duality. The rest of this section will be devoted to analysing this question.

We first remind the reader of the familiar story in type IIB. Consider, for instance, the intersection of two D5-branes on a string. The open strings from one brane to the other give rise to chiral fermions living on the intersection. These chiral fermions lead to gauge and gravitational anomalies, which are cancelled by anomaly inflow from the branes into the intersection [1] in]. For this to happen, the D5-branes need, amongst other terms, a

$$
C_{2} \wedge p_{1}
$$

coupling in their Wess-Zumino action, where $C_{2}$ is the $\mathrm{R}-\mathrm{R}$ two-form potential and $p_{1}$ the first Pontrjagin class of the tangent bundle of the D5brane world-volume. Now, type IIB S-duality implies the presence of chiral fermions on the intersection of two NS5-branes on a string, and thus a

$$
B_{2} \wedge p_{1}
$$

term on the world-volumes of each NS5-brane ( $B$ is the NS-NS two-form). Indeed, the following argument ${ }^{2}$ for the presence of this term was given in Ref. [120]. Under T-duality in a transverse direction the NS5-brane is mapped to a KaluzaKlein monopole in type IIA. This object is described by a Euclidean Taub-NUT metric in the transverse space. Since $p_{1}(\mathrm{ETN}) \neq 0$ and the $B_{2} \wedge X_{8}$ term discussed in the previous section contains a piece proportional to $B_{2} \wedge\left(p_{1}\right)^{2}$, reducing this term gives rise to the required $B_{2} \wedge p_{1}$ term on the IIA Kaluza-Klein monopole and thus on the IIB NS5-brane.

What about type 0B? It has been argued [1] 10 in that chiral fermions and thus anomalies are present on the intersection of electric and magnetic D5-branes. Hence, type 0 D5-branes have the $p_{1}$ couplings in their Wess-Zumino actions (this has also been checked via a string computation $\left[10_{1}^{\prime \prime}\right)$. We expect that the number of chiral fermion zero modes on these intersections cannot change under a continuous increase of the string coupling. On the other hand, we have argued that there is no $B_{2} \wedge X_{8}$ term in type $0 \mathrm{~A}$, so we expect no $B_{2} \wedge p_{1}$ coupling of type 0B NS5branes, and thus no chiral fermions on intersections of NS5-branes. If this reasoning is valid, it could be a problem for type 0B S-duality.

\section{A little string interpretation}

In type II string theories the fivebrane worldvolume theories have been conjectured to be properly described by "little strings" [1 1 in 3 ". These noncritical closed string theories have hitherto remained mysterious, although some qualitative features may be understood. We briefly recall some of these, focusing on the type IIB case. However, we prefer first to treat the more familiar example of a fundamental string, providing a guiding reference when dealing with the little strings.

A fundamental string solution in supergravity breaks half of the 32 bulk supersymmetries. These broken symmetries give rise to fermion

\footnotetext{
${ }^{2}$ In fact, this is how the $C_{2} \wedge p_{1}$ coupling on D5-branes was first discovered.
} 
zero modes on the string, of which 8 are leftmoving and 8 right-moving. They are identified with the Green-Schwarz space-time fermion fields on the world-volume. Upon quantization of these fermions 128 bosons and 128 fermions are found, together building the on-shell $\mathcal{N}=2$ supergravity multiplet. This multiplet is also found from the RNS superstring, so the picture is consistent. In the latter formulation it is not hard to see that the NS-NS sector fields together with, say, the RNS sector build an $\mathcal{N}=1$ supergravity multiplet. Likewise, the NS-R and R-R sector are combined into one multiplet of $\mathcal{N}=1$.

Let us move on now to little strings. One way to derive properties of little strings in type IIB is to consider a gauge instanton on a D5 brane. Half of the bulk supersymmetries are unbroken by the D5-brane. Sixteen real supercharges are thus found, obeying a $\mathcal{N}=(1,1)$ superalgebra. Of these, half are broken by the instanton. On the macroscopic string there are thus eight fermionic modes, four left-moving and four right-moving ones. Upon quantization of these zero modes one finds 8 bosonic and 8 fermionic states. They correspond to the on-shell degrees of freedom of one $\mathcal{N}=(1,1)$ vectormultiplet. ${ }^{3}$ Upon decomposition with respect to $\mathcal{N}=1$ six-dimensional supersymmetry one hypermultiplet and one vectormultiplet result. It is conceivable that a similar analysis in type IIA, if possible at all, would give a $\mathcal{N}=(2,0)$ tensormultiplet, decomposing into one hypermultiplet and one tensormultiplet of $\mathcal{N}=1$ supersymmetry. We remark that the tensormultiplet has a bosonic content that consists of one scalar and one two-form potential with self-dual field strength. All in all, one may wish to draw the following analogies between little strings and type II strings. In both cases there is one universal sector, the hypermultiplet resp. the (NS-NS + R-NS) sector. The other sector depends on whether or not the supersymmetry in the "bulk" is chiral, where "bulk" means the fivebrane worldvolume for the little string and ten-dimensional spacetime for the type II string. For the nonchiral type IIA and little type iia the remaining bosonic fields are odd $q$-form potentials, cou-

\footnotetext{
${ }^{3}$ Since the little strings of type IIB are non-chiral, we will name them type iia little strings.
}

pling to even $p \mathrm{D} p$ - resp. $\mathrm{d} p$-branes. As to chiral type IIB/iib the R-R sector has only even $q$ form potentials, of which one has a self-dual field strength. In these theories only odd $p \mathrm{D} p$ or $\mathrm{d} p$ branes occur. So this analogy, however formal, is at least remarkable.

As to the various fivebranes, the type IIA NS fivebrane theory is conjectured to be described by chiral iib little strings, whereas iia little strings would do the job for type IIB fivebranes.

If one is willing to take the above for granted, one may then proceed and construct "little type 0a/b strings" by mimicking the procedure for the bulk strings: spacetime fermions are removed and there is a doubled R-R spectrum compared to the superstring. Concretely this would imply that the massless spectrum of little type 0a strings consists of the bosonic contents of one hypermultiplet and two vectormultiplets, in $\mathcal{N}=1$ language. This precisely matches with the spectrum on the type 0B NS fivebrane. Little type 0b strings would then generate the bosonic content of one hypermultiplet, one selfdual tensormultiplet and one anti-selfdual tensormultiplet. Also here this seems to be consistent with the spectrum of the type 0A NS fivebrane.

\section{Conclusions}

From a zero mode analysis of type 0 KaluzaKlein monopoles, we have derived the massless degrees of freedom of NS5-branes in type 0A and 0B. The massless spectrum is purely bosonic and non-chiral in both cases. The 0A NS5-brane contains an unrestricted tensor (rather than a selfdual one) whereas two gauge fields live on the $0 \mathrm{~B}$ NS5-brane.

Since the spectrum of a 0A NS5-brane is nonchiral, the anomaly-cancelling one loop bulk term present in type IIA should be absent in type 0A. This is indeed the case.

We have pointed out some puzzles concerning type $0 \mathrm{~B}$ S-duality. In particular, the question is raised whether the absence of a $B_{2} \wedge X_{8}$ term in the bulk of type 0A makes the duality of NS5branes with D5-brane pairs impossible.

It is tempting to interpret the spectrum we find in terms of "type 0 little strings". Their relation to the usual type II "little strings" is very 
similar of the relation between the bulk type 0 and type II strings: fermions are projected out and the "Ramond-Ramond" spectrum is doubled.

\section{Acknowledgments}

We thank the organizers for a very nice conference. B.C thanks them also for the opportunity to present this work.

\section{References}

[1]_B. Craps and F. Roose, 'J. High Energy Phys.', - $10(1999)$ 007, hep-th/9906179'

[2] _L.J. Dixon and J.A. Harvey, 'Nucl. Phys. B 274, $(1986)-93$

[3] O. Bergman and M.R. Gaberdiel, J. High En-4 '- - _ ergy Phys. 07_(1999)_022, hep-th/9906055'.

[4] C.G. Callan, J.A. Harvey and A. Strominger, INucl. Phys. B 367 (1991) 60.

_[5] C.G. Callan and J.A. Harvey, 'Nucl. Phys. B' - - 250 (1985) 427!.

[6]_ M.J. Duff, J.T._Liu and R. Minasian, 'Nucl.' 1. - Phys. B 452 (1995) 261, hep-th/9506126 E. Witten, 'Nucl. Phys. B 463 (1996) 383i, hep-th/9512219.

[7] C. Vafa and E. Witten, 'Nucl. Phys. B $\mathbf{4} \overline{4} \overline{7}$, ; $(1995) 261$, hep-th/9505053'.

[8] I.R. Klebanov and A.A. Tseytlin, 'Nucl. Phys. B' - _ $\mathbf{5} \mathbf{4} \mathbf{6}(1999)$ 155, hep-th/9811035.

[9] O. Bergman and M.R. Gaberdiel, 'Nucl. Phys.' ' - _ B 499 (1997) 183, hep-th/9701137.

[10] M. Billó, B. Craps and F. Roose, iPhys. Lett. Bi 457 (1999) 61, hep-th/9902196'.

[11] M. Green, J. Harvey and G. Moore, Class. Quant. Grav. 14 (1997) 47, hep-th/9605033.

[12] M. Bershadsky, V. Sadov and C. Vafa, 'Nucl.' ' - _ Phys. B $\mathbf{4 6 3}(1996)$ 420, hep-th/9511222!

[13] N. Seiberg, iPhys. Lett. B _ 408 (1997) 98; hep-th/9705221. 